\title{
A NOTE ON REAL HYPERSURFACES OF A COMPLEX PROJECTIVE SPACE
}

\author{
JUNG-HWAN KWON and HISAO NAKAGAWA
}

(Received 24 August 1987; revised 22 March 1988)

Communicated by K. Mackenzie

\begin{abstract}
We study real hypersurfaces of a complex projection space and show that there are no such hypersurfaces with harmonic curvature on which the structure vector is principal.

1980 Mathematics subject classification (Amer. Math. Soc.) (1985 Revision): 53 B 25, 53 C 40.

\section{Introduction}

Let $P^{n} C$ be an $n$-dimensional complex projective space with Fubini-Study metric of constant holomorphic sectional curvature 4. Let $M$ be a real hypersurface of $P^{n} C$ and $(P, E, \omega, g)$ be an almost contact metric structure induced from the complex structure of $P^{n} C$. Kimura [2] proved recently the following
\end{abstract}

THEOREM A. There are no real hypersurfaces with parallel Ricci tensor of $P^{n} C$ on which $E$ is principal.

The hypersurface $M$ is said to be with harmonic curvature, if the Ricci tensor $S$ satisfies

$$
\nabla_{X} S(Y)=\nabla_{Y} S(X)
$$

The first author was partially supported by KOSEF and the second author was partially supported by JSPS and KOSEF.

(C) 1989 Australian Mathematical Society 0263-6115/89 \$A2.00+0.00 
for any vector fields $X$ and $Y$, where $\nabla$ denotes the Riemannian connection of $M$. The purpose of this note is to prove the following

THEOREM. There are no real hypersurfaces with harmonic curvature of $P^{n} C$ on which $E$ is principal.

The authors would like to express deep thanks to the referee for his criticism and suggestion.

\section{Preliminaries}

Let $M$ be a real hypersurface of $P^{n} C(n \geq 2)$. On a neighborhood of each point, by $\xi$ is denoted a local unit normal vector field of $M$ in $P^{n} C$. As is well known, $M$ admits an almost contact metric structure induced from the complex structure $J$ of $P^{n} C$ (see Yano and Kon [5]). Namely, for the Riemannian metric $g$ of $M$ induced form the Fubini-Study metric $g^{\prime}$ of $P^{n} C$, we define a tensor field $P$ of type $(1,1)$, a vector field $E$ and a 1 -form $\omega$ on $M$ by

$$
g(P X, Y)=g^{\prime}(J X, Y), \quad g(E, Y)=\omega(Y)=g^{\prime}(J \xi, Y)
$$

for any vector fields $X$ and $Y$ on $M$. Then we have

$$
P^{2} X=-X+\omega(X) E, \quad P E=0, \quad g(E, E)=1 .
$$

Moreover we have

$$
g(P X, P Y)=g(X, Y)-\omega(X) \omega(Y) .
$$

By $\nabla$ and $\nabla^{\prime}$ are denoted the Riemannian connections of $M$ and $P^{n} C$ respectively. The Gauss and Weingarten formulas are given by

$$
\nabla_{X}^{\prime} Y=\nabla_{X} Y+g(A X, Y) \xi
$$

and

$$
\nabla_{X}^{\prime} \xi=-A X,
$$

respectively, where $A$ is the shape operator of $M$ in $P^{n} C$ derived from the unit vector $\xi$. From (1.3) it follows easily that we have

$$
\nabla_{X} P(Y)=\omega(Y) A X-g(A X, Y) E,
$$

and

$$
\nabla_{X} E=P A X
$$


Let $R$ be the Riemannian curvature tensor of $M$. Since $P^{n} C$ is of constant holomorphic sectional curvature 4, we have the following Gauss and Codazzi equations

$$
\begin{gathered}
R(X, Y) Z=g(Y, Z) X-g(X, Z) Y+g(P Y, Z) P X-g(P X, Z) P Y \\
-2 g(P X, Y) P Z+g(A Y, Z) A X-g(A X, Z) A Y \\
\nabla_{X} A(Y)-\nabla_{Y} A(X)=\omega(X) P Y-\omega(Y) P X-2 g(P X, Y) E
\end{gathered}
$$

By (1.1), (1.6), (1.7) and (1.8) we get

$$
S X=(2 n+1) X-3 \omega(X) E+h A X-A^{2} X,
$$

and

$$
\begin{aligned}
\nabla_{X} S(Y)= & -3\{g(P A X, Y) E+\omega(Y) P A X\} \\
& +d h(X) A Y+(h-A) \nabla_{X} A(Y)-\nabla_{X} A(A Y),
\end{aligned}
$$

where $h=\operatorname{Tr} A$ and $S$ denotes the Ricci tensor of $M$.

An eigenvector $X$ of the shape operator $A$ is called a principal vector and an eigenvalue $\lambda$ is called a principal curvature. We assume that structure vector $E$ is principal. By $\alpha$ is denoted the principal curvature associated with $E$, that is, it satisfies $A E=\alpha E$. Then it is seen that $\alpha$ is constant (see [5]) and hence (1.6) implies $\nabla_{X} A(E)=\alpha P A X-A P A X$, from which, together with the Codazzi equation (1.8), it follows that

$$
\begin{aligned}
2 A P A & =\alpha(P A+A P)+2 P, \\
\nabla_{X} A(E) & =\alpha(P A-A P) X / 2-P X, \text { and } \\
\nabla_{E} A(Y) & =\alpha(P A-A P) Y / 2 .
\end{aligned}
$$

\section{Proof of theorem}

First of all, we define a tensor field $T$ of type $(0,3)$ by

$$
T(X, Y, Z)=g\left(\nabla_{X} S(Y)-\nabla_{Y} S(X), Z\right)
$$

for any vector fields $X, Y$ and $Z$ on $M$. According the hypersurface $M$ has harmonic curvature if and only if the tensor field $T$ vanishes identically. By means of $(1.10)$, we have

$$
\begin{aligned}
T(X, Y, Z)= & \omega(X)\{h g(P Y, Z)-g((A P-3 P A) Y, Z)\} \\
& -\omega(Y)\{h g(P X, Z)-g((A P-3 P A) X, Z)\} \\
& -\omega(Z)\{2(h-\alpha) g(P X, Z)+3 g((P A+A P) X, Y)\} \\
& +d h(X) g(A Y, Z)-d h(Y) g(A X, Z) \\
& +g\left(A X, \nabla_{Y} A(Z)\right)-g\left(A Y, \nabla_{X} A(Z)\right) .
\end{aligned}
$$


Assume that $M$ has harmonic curvature. Taking account of the second equation of (1.11) and (2.1) with $Z=E$, we have

$$
\begin{array}{r}
-2 g((P A+A P) X, Y)-\alpha g(A P A X, Y)+\alpha g\left(\left(P A^{2}+A^{2} P\right) X, Y\right) / 2 \\
+2(\alpha-h) g(P X, Y)+\alpha\{d h(X) \omega(Y)-d h(Y) \omega(X)\}=0 .
\end{array}
$$

Similarly, putting $X=E$ in (2.2), we obtain

$$
\begin{aligned}
& g((3 P A-A P) Y, Z)+(h-\alpha) g(P Y, Z)+\alpha^{2} g((P A-A P) Y, Z) / 2 \\
& \quad-\alpha g((P A-A P) A Y, Z) / 2+d h(E) g(A Y, Z)-\alpha d h(Y) \omega(Z)=0
\end{aligned}
$$

and then putting $Z=E$, we have

$$
\alpha\{d h(E) \omega-d h\}=0 .
$$

Accordingly, from (2.3) and (2.5) it follows that

$$
\begin{aligned}
& T(X, Y, E)+\omega(Y) T(E, X, E)-\omega(X) T(E, Y, E) \\
& =\alpha g\left(\left(P A^{2}+A^{2} P\right) X, Y\right) / 2-2 g((P A+A P) X, Y)-\alpha g(A P A X, Y) \\
& \quad+2(\alpha-h) g(P X, Y)=0 .
\end{aligned}
$$

Therefore (2.4) and the above equation mean that if $M$ has harmonic curvature, then we have

(2.6) $3 P A-A P+(h-\alpha) P+\alpha(P A-A P)(\alpha-A) / 2+\beta A-\alpha \operatorname{grad} h \otimes \omega=0$,

and

$$
\alpha\left(P A^{2}+A^{2} P\right) / 2-2(P A+A P)-\alpha A P A+2(\alpha-h) P=0,
$$

where $\beta=d h(E)$.

We prove here that the principal curvature $\alpha$ is a non-zero constant. Suppose that $\alpha=0$. Then (2.6) and (2.7) are reduced to $3 P A-A P+h P+\beta A=0$, $P A+A P+h P=0$, and hence we have $4 P A+2 h P+\beta A=0$. let $X$ be a principal vector with principal curvature $\lambda$ which is orthogonal to $E$. Then, by means of the above equation, we have $(4 \lambda+2 h) P X+\beta \lambda X=0$, which implies that $4 \lambda+2 h=0$ and $\beta \lambda=0$, because $X$ and $P X$ are mutually orthogonal. This yields that the trace of $A$ satisfies $h=\alpha+(2 n-2) \lambda=-(n-1) h$, which means that $\lambda=h=0$, and hence $M$ is totally geodesic, a contradiction.

Next, the fact that $h$ is constant is proved. Since $\alpha$ is non-zero constant, (2.5) yields $\operatorname{grad} h=\beta E$ or $d h=\beta \omega$, from which we have $d \beta(X) \omega(Y)-$ $d \beta(Y) \omega(X)=-\beta g((P A+A P) X, Y)$, because of the fact that

$$
g\left(\nabla_{X} \operatorname{grad} h, Y\right)=g\left(\nabla_{Y} \operatorname{grad} h, X\right) .
$$

Suppose that there exist points $x$ at which $\beta(x) \neq 0$. Putting $Y=E$ in the above equation we have $d \beta=d \beta(E) \omega$ and hence this implies that 
$\beta(P A+A P)=0$, which contradicts the first equation of (1.11). Thus $\beta$ vanishes identically and by $(2.5), h$ must be constant.

For a principal vector $X$ with principal curvature $\lambda$ which is orthogonal to $E, Y=P X$ is also a principal vector with principal curvature $\mu=$ $(\alpha \lambda+2) /(2 \lambda-\alpha)$, by the first equation of (1.11). Hence (2.6) gives rise to

$$
3 \lambda-\mu+h-\alpha+\alpha(\lambda-\mu)(\alpha-\lambda) / 2=0,
$$

because $h$ is constant. Accordingly the principal curvature $\lambda$ is the root of the following cubic equation with constant coefficients

$$
\alpha x^{3}-2\left(\alpha^{2}+3\right) x^{2}+\left(\alpha^{3}+5 \alpha-2 h\right) x+(\alpha h+2)=0 .
$$

Thus $M$ has at most four distinct constant principal curvatures. By Kimura's theorem [1], $M$ is congruent to an open subset of a homogeneous real hypersurface of type $A_{1}, A_{2}$ or $B$ of $P^{n} C$.

On the other hand, for a principal vector $Y=P X$ with principal curvature $\mu, P Y=-X$ is also a principal vector with principal curvature $\lambda$ and hence we can change $\lambda$ and $\mu$ in (2.8). Thus we have $3 \mu-\lambda+h-\alpha+\alpha(\mu-\lambda)(\alpha-\mu) / 2=0$, which together with $(2.8)$ yield $(\lambda-\mu)\left\{\alpha(\lambda+\mu)-2\left(\alpha^{2}+4\right)\right\}=0$. this is equivalent to $\left(\lambda^{2}-\alpha \lambda-1\right)\left\{\alpha \lambda^{2}-2\left(\alpha^{2}+4\right) \lambda+\alpha\left(\alpha^{2}+5\right)\right\}=0$.

Suppose that $M$ is congruent to an open subset of a homogeneous real hypersurface of type $B$. Then the distinct principal curvatures at three, say $\alpha=2 \cot 2 t, \lambda_{1}=\cot (t-\pi / 4)$ and $\lambda_{2}=-\tan (t-\pi / 4)$ (for details, see [4, page 47 , Table]). By the way, $\lambda_{1}$ and $\lambda_{2}$ have to satisfy $\lambda^{2}-2(\cot 2 t) \lambda-1=0$, which leads to a contradiction. Thus $M$ is congruent to an open subset of a homogeneous hypersurface of type $A_{1}$ or $A_{2}$. By a theorem in [3], the Ricci tensor $S$ is cyclic-parallel, namely it satisfies

$$
g\left(\nabla_{X} S(Y), Z\right)+g\left(\nabla_{Y} S(Z), X\right)+g\left(\nabla_{Z} S(X), Y\right)=0 .
$$

hence it is parallel and we can apply Theorem $A$ to our situation, which concludes the proof.

\section{References}

[1] M. Kimura 'Real hypersurfaces and complex submanifolds in complex projective space', Trans. Amer. Math. Soc. 296 (1986), 137-149.

[2] M. Kimura, 'Real hypersurfaces of a complex projective space' Bull. Austral. Math. Soc. 33 (1986), 383-387.

[3] J.-H. Kwon and H. Nakagawa, 'Real hypersurfaces with cyclic-parallel Ricci tensor of a complex projective space', Hokkaido Math. J. 17 (1988), 355-371. 
[4] R. Takagi, 'Real hypersurfaces in a complex projective space with constant principal curvatures', J. Math. Soc. Japan 27 (1975), 43-53.

[5] K. Yano and M. Kon, 'CR submanifolds of Kaehlerian and Sasakian manifolds', Birkhäuser, 1983.

Department of Mathematics,

Institute of Mathematics

Taegu University

Taegu, 705-033

University of Tsukuba

Korea Ibaraki, 305

Japan 